\title{
A systems biology approach to suppress TNF-induced proinflammatory gene expressions
}

\author{
Kentaro Hayashi ${ }^{1,2}$, Vincent Piras ${ }^{1,2}$, Sho Tabata ${ }^{1,2}$, Masaru Tomita ${ }^{1,2}$ and Kumar Selvarajoo ${ }^{1,2^{*}}$
}

\begin{abstract}
Background: Tumor necrosis factor (TNF) is a widely studied cytokine (ligand) that induces proinflammatory signaling and regulates myriad cellular processes. In major illnesses, such as rheumatoid arthritis and certain cancers, the expression of TNF is elevated. Despite much progress in the field, the targeted regulation of TNF response for therapeutic benefits remains suboptimal. Here, to effectively regulate the proinflammatory response induced by TNF, a systems biology approach was adopted.

Results: We developed a computational model to investigate the temporal activations of MAP kinase (p38), nuclear factor (NF)-KB, and the kinetics of 3 groups of genes, defined by early, intermediate and late phases, in murine embryonic fibroblast (MEF) and 3 T3 cells. To identify a crucial target that suppresses, and not abolishes, proinflammatory genes, the model was tested in several in silico knock out $(\mathrm{KO})$ conditions. Among the candidate molecules tested, in silico RIP1 KO effectively regulated all groups of proinflammatory genes (early, middle and late). To validate this result, we experimentally inhibited TNF signaling in MEF and 3T3 cells with RIP1 inhibitor, Necrostatin-1 (Nec-1), and investigated 10 genes (II6, Nfkbia, Jun, Tnfaip3, CCl7, Vcam1, CxCl10, Mmp3, Mmp13, Enpp2) belonging to the 3 major groups of upregulated genes. As predicted by the model, all measured genes were significantly impaired.
\end{abstract}

Conclusions: Our results demonstrate that Nec-1 modulates TNF-induced proinflammatory response, and may potentially be used as a therapeutic target for inflammatory diseases such as rheumatoid arthritis and osteoarthritis.

Keywords: TNF, Cell signaling, Computational model, Inflammation, RIP1

\section{Introduction}

The tumor necrosis factor (TNF), first termed in 1962 [1], was initially known for its ability to induce programmed cell death or apoptosis. As a result, throughout the years, the TNF has been intensely investigated for its anticancer property [2]. Today, this cytokine is central to the regulation of myriad important cellular processes such as proliferation, differentiation, growth, and the immune response.

TNF binds to two types of outer membrane bound receptors on target cells, TNFR1 and TNFR2, and triggers the cell survival and proinflammatory NF- $\mathrm{KB}$ and MAP kinases activations [3]. In addition, the TNFR1 induces intracellular cell death pathways via caspases after internalization through endocytosis. It is, therefore, conceivable that the dysregulation of the TNF signaling process will

\footnotetext{
* Correspondence: kumar@ttck.keio.ac.jp

${ }^{1}$ Institute for Advanced Biosciences, Keio University, 14-1 Baba-cho, Tsuruoka, Japan

${ }^{2}$ Systems Biology Program, Graduate School of Media and Governance, Keio University, Fujisawa, Endo 5322, Japan
}

misbalance proinflammatory and/or apoptotic responses. Notably, the chronic aberration in the baseline levels of TNF in human circulatory system has been attributed to the pathogenesis of numerous diseases, including rheumatoid arthritis, osteoporosis, sepsis and cancer $[4,5]$.

The vast majority of TNF related biological processes are initiated by the death-domain (DD) containing TNFR1, which is also called TNFRSF1A. Unlike TNFR2, TNFR1 is present in almost all cell types in humans. Upon TNF binding, TNFR1 trimerizes, and its intracellular DD recruits TRADD, which then creates a platform for RIP1 and TRAF2 to collectively form the receptor-signaling complex I. Cellular inhibitor of apoptosis proteins (cIAP)1 and -2 bind to complex I and, consequently, together with K63-linked ubiquitin chains, modify RIP1 and TRAF2 [6]. This creates docking sites for an E3 ligase or linear ubiquitin chain assembly complex (LUBAC) consisting of heme-oxidized IRP2 ubiquitin ligase-1 (HOIL-1), HOIL-1-interacting protein (HOIP), and SHANKassociated RH domain interacting protein (SHARPIN). 
Subsequently, the activation of TAK1 and the ubiquitination of NEMO (or IKK $\gamma$ ), a subunit of IKK complex, lead to cell survival or proinflammatory response through NF- $k B$ and MAP kinases activations. Other TRAF superfamily members (TRAF5 and 6) are also known to play a role in the NF- $\mathrm{kB}$ and MAP kinases activations $[7,8]$.

On the other hand, for the apoptotic pathways, clathrin, AP-2 and Dyn first mediate receptor internalization. Receptor-signaling complex I becomes modified, and dissociates from TNFR1, allowing FADD and caspase- 8 to form complex II. Within complex II, caspase- 8 becomes activated to induce extrinsic apoptosis through caspase- 3 activation. Alternatively, caspase- 8 activates caspase- 7 , and eventually, the cleavage of Bid to tBid in the mitochondria activates caspase- 9 via cathepsin D. This induces the intrinsic apoptosis through caspase-3 activation.

Due to its ability to signal numerous cellular processes via the survival and death pathways, the TNFR1 signaling research has received immense attention over the years, especially on understanding the downstream signaling cascades to regulate and control proinflammatory diseases and cancer. Despite numerous studies, the control of proinflammatory diseases through therapeutic treatments, where TNF is over-expressed, remains suboptimal. For example, biologic response modifiers or biologics, such as Etanercept and Infliximab, are TNF decoy receptors or antibodies that suppress TNFR1 signaling through competition for TNF. Although these drugs have shown successful downregulation of inflammation in many cases, they can immuno-compromise patients to secondary infections such as tuberculosis [9], or have been ineffective in a substantial number of administered patients [10].

To find alternatives, there have been major efforts on selectively suppressing the intracellular signaling of TNFR1. For example, genetic knockouts (KOs) of TRAFs and TRADD acting on the proinflammatory pathways have been investigated $[7,8,11]$. However, the experimental outcomes, so far, have not been optimistic. In TRAF2 KO, there is compensatory activation of NF- $\mathrm{kB}$ through TRAF5 [7] or TRAF6 [8], and vice-versa. On the other hand, TRADD KO almost completely abolishes NF-кB activation [11], which is not desirable for the general survivability of cells. Thus, a systemic approach where the propagation of signal transduction to all known branching pathways during target intervention should be monitored. This will allow the elucidation of effective target candidate(s) that overcomes and balances the deficiencies of current investigations.

In this paper, we adopted a systems biology approach to study TNFR1 signaling dynamics. Firstly, we developed a computational model of TNF-induced proinflammatory response leading to NF- $\mathrm{B}$, MAP kinase activations, and three groups of gene expressions (classified according to their temporal profiles [12]). The model is based on the perturbation-response approach [13-16], which has been successfully used to elucidate novel signaling features and behaviors in Toll-like receptor-4 [17,18], -3 [19], and TNFrelated apoptosis-inducing ligand (TRAIL) signaling [20]. Secondly, the TNFR1 model parameters were selected to fit the temporal activation profiles of NF- $\mathrm{kB}$ and MAP kinase p38 for fibroblast cell type in several available conditions (wildtype [7], TRAF2 KO [7], TRAF5 KO [7], TRAF2/ TRAF5 double KO (DKO) [7], TRAF6 KO [8], TRADD KO [11] and RIP1 KO [21]). Using the resultant TNFR1 model with robust parameters, we performed simulations of multiple in silico KOs to determine an optimal target that suppresses, but not abolishes, proinflammatory genes. Finally, to validate the modeling results, we performed experiments measuring various key proinflammatory gene expressions in MEF and 3T3 cells for TNF stimulation. Overall, our study presents evidence that systems biology research can be useful to elucidate important target(s) to suppress proinflammatory diseases such as rheumatoid arthritis and osteoarthritis.

\section{Results}

\section{TNFR1 signaling topology and model}

To develop a computational model of proinflammatory TNFR1 signaling dynamics, we first require the known signal transduction pathways. We curated the KEGG database, and performed literature survey of the latest TNF research. After carefully considering several sources, we were able to propose a signaling topology mainly by combining the knowledge from KEGG, Falschlehner et al. (2012) and Wertz et al. (2010) [6,22] (Figure 1).

Next, to simulate TNF-induced dynamics of NF- $\mathrm{kB}$ and MAPK activations using the topology, we developed a dynamic model based on perturbation-response approach (Materials and Methods), using COPASI simulation platform [23]. Unlike common biochemical reaction models [24,25], the perturbation-response approach does not require detailed knowledge of all signaling species and their reaction kinetics. This is because it analyses the response waves of signal transduction instead of individual reaction kinetics [13-15,17-20]. The response waves can be approximated using linear response rules (Response Rules, Additional file 1: Figure S1) combined with the law of mass conservation, and this approach has been previously used to successfully model the TLRs and TRAIL signaling pathways [17-20].

Briefly, each reaction in the model is represented by a first-order response equation with activation or deactivation term. The activation term generally refers to protein binding, transformation, complex formation, phosphorylation and transcription. The deactivation term refers to protein unbinding, dephosphorylation and negative regulation such as mRNA decay through microRNA regulation. 


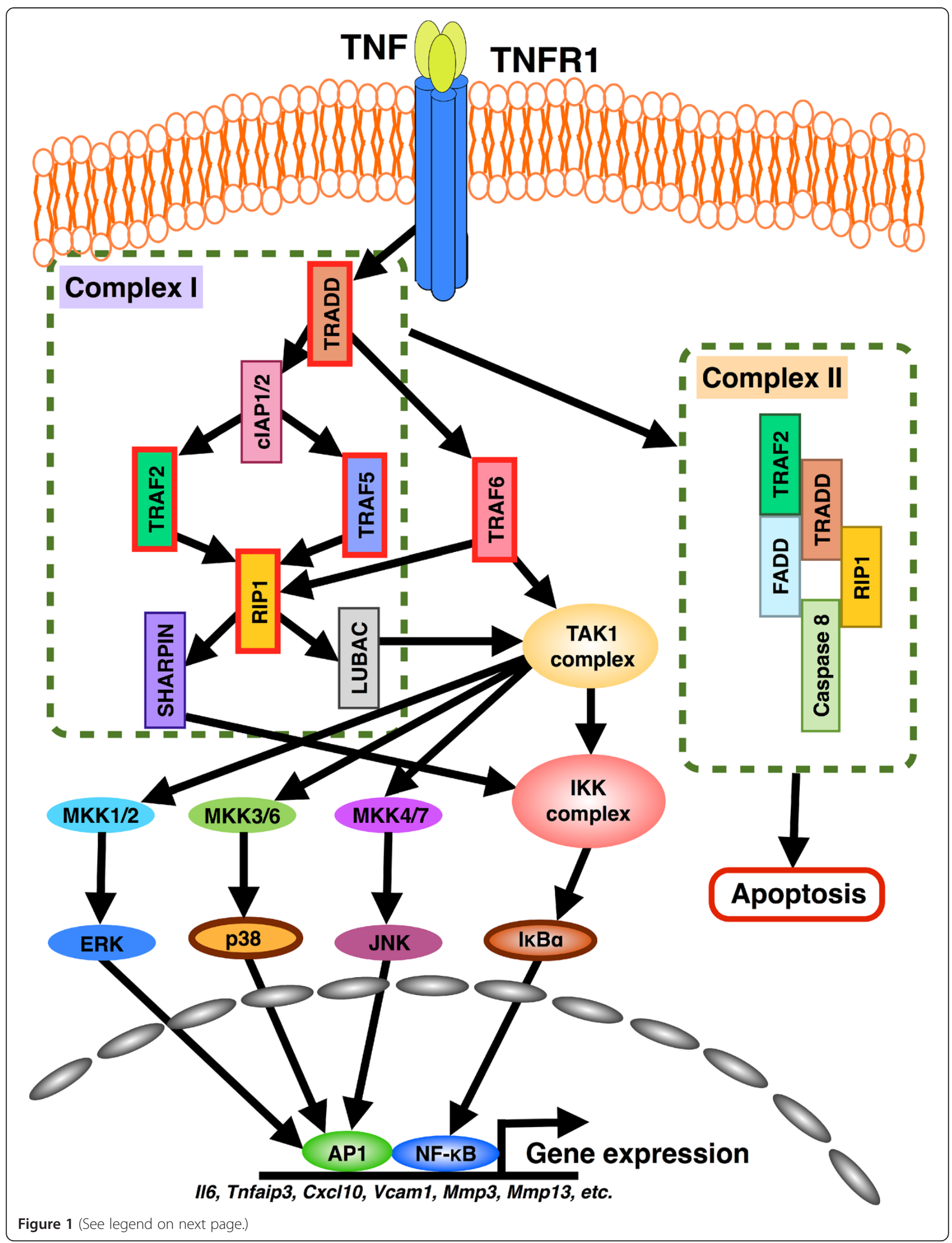


(See figure on previous page.)

Figure 1 Schematic of TNFR1 signaling of cell survival/proinflammatory and apoptosis pathways. Upon TNF receptor activation, complexes I (survival pathway) and II (apoptosis) are formed. Complex I subsequently activates transcription factors, such as activator protein (AP)-1 and NF-KB through MAP kinases and IKK complex, respectively, which subsequently bind to promoter regions of genes to induce numerous proinflammatory genes.

Simulating TNF-induced NF-KB and MAP kinase dynamics The parameters of the initial model (rate constants, or the elements of Jacobian matrix $\boldsymbol{J}$, Materials and Methods) were estimated by fitting the simulation profiles with experimental profiles of signaling molecules where data is available. We obtained published semi-quantitative experimental profiles of $\mathrm{I} \kappa \mathrm{B} \alpha$ phosphorylation (NF- $\mathrm{KB}$ activation) and p38 (MAP kinase) activation in wildtype and various genetically mutant MEFs generally treated with $10 \mathrm{ng} / \mathrm{mL}$ of TNF (Figure 2A, Additional file 1: Figure S2 and Table S1) $[7,8,11,21]$. (Note that the kinetics of other MAP kinases, JNK and ERK, were also similar to p38 $[7,8,11,21]$. Thus, we used p38 as a representative MAP kinase for our investigation).

The parameter values were selected by using Genetic Algorithm [26] module in COPASI software [23] to fit the experimental profiles (Figure 2A, WT). Following, we performed sensitivity analysis (Materials and Methods) of the model parameters and found them to be robust to a small degree of uncertainty to their values (Additional file 1: Table S2). As a further validity of the parameter values, we tested the wildtype model in other conditions, namely TRAF2 KO, TRAF5 KO, TRAF2/5 double KO, TRAF6 KO, RIP1 KO and TRADD KO (Figure 2B). (Note that in silico KOs were generated from the wildtype model by setting the activation parameter value of the KO molecule to null).

Remarkably, we were able to obtain a single set of model parameters (Table 1, reactions 1-29 and see Additional file 2 for the TNFR1 model A in SBML format), which could be used to simulate the semi-quantitative profiles of IkB $\alpha$ phosphorylation and p38 kinase activation in multiple experimental conditions. In wildtype, TRAF2 KO, TRAF5 $\mathrm{KO}$ and TRAF6 $\mathrm{KO}$, the $\mathrm{IkB} \alpha$ phosphorylation and p38 kinase activation reach peak values around $15 \mathrm{~min}$ and gradually decay at $30 \mathrm{~min}$. Notably, TRAF6 KO shows
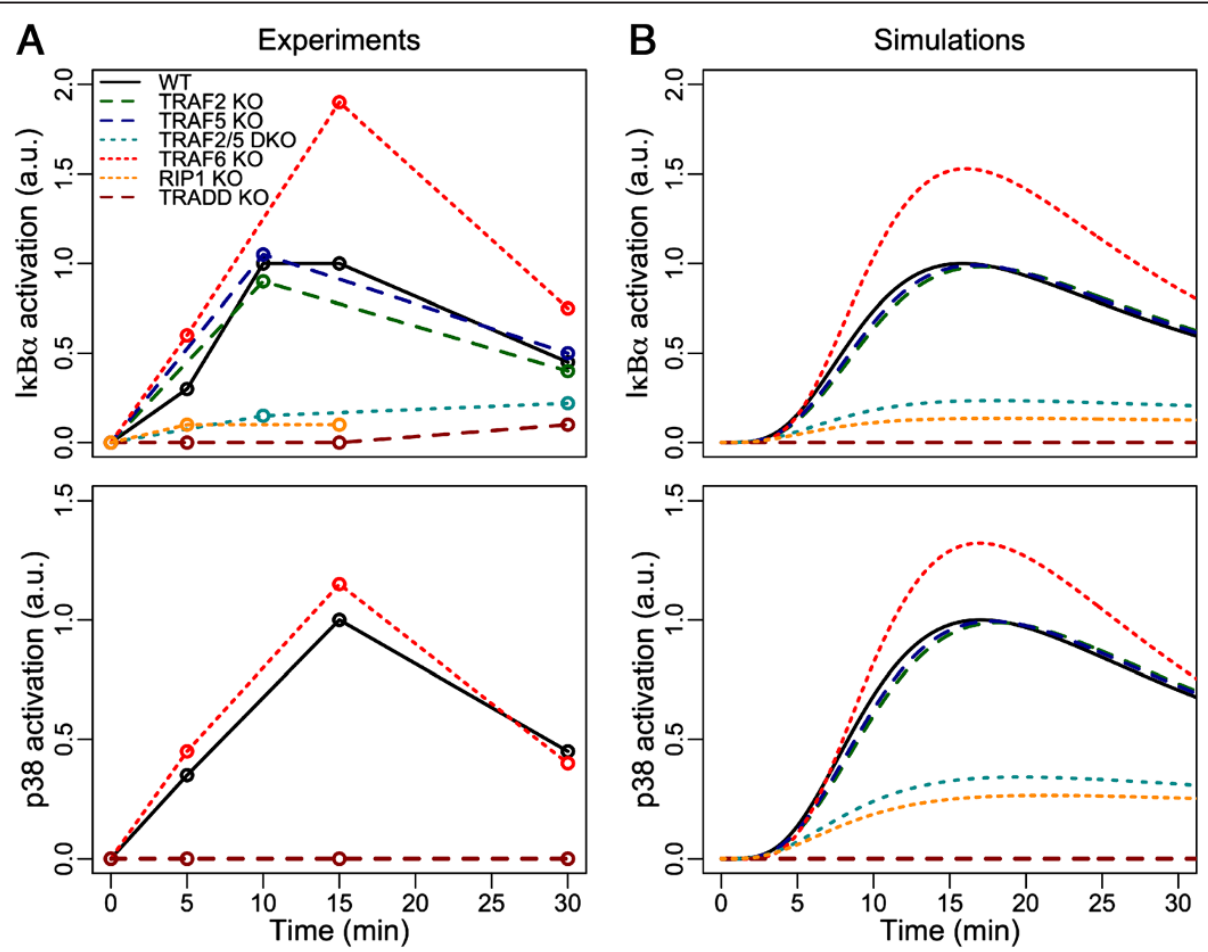

Figure 2 Experimental and simulated profiles of $\mathrm{IKBa}$ and p38 activations in wildtype and mutant conditions. (A) Experimental profiles, MEFs were generally treated with $10 \mathrm{ng} / \mathrm{mL}$ of TNF, and (B) simulated profiles of $1 \mathrm{kBa}$ (top panels) and p38 (bottom panels) activations up to 30 min in wildtype (WT), TRAF2 KO, TRAF5 KO, TRAF2/TRAF5 double KO (TRAF2/5 DKO), TRAF6 KO, TRADD KO and up to 15 min in RIP1 KO. Note: p38 experimental profiles are available only for WT, TRAF6 KO and TRADD KO. Experimental details and data are found in references [7,8,1 1,21]. ImageJ was used to estimate the intensities of the activation dynamics (Additional file 1: Table S1) for each molecule in each condition relative to wildtype peak activation values found in Additional file 1: Figure S2. IKBa phosphorylation refers to NF-KB activation throughout the text. 
Table 1 TNFR1 model A

\begin{tabular}{|c|c|c|c|c|c|c|c|c|c|c|}
\hline \multirow[b]{2}{*}{$1^{1}$} & \multicolumn{3}{|c|}{ Reaction } & \multicolumn{6}{|c|}{ Formula and parameters $\left(\mathrm{s}^{-1}\right)$} & \multirow{2}{*}{$\begin{array}{r}\text { Remarks } \\
\text { Activation of TRADD by TNFR1 }\end{array}$} \\
\hline & TNFR1 & $\rightarrow$ & TRADD & $k_{1}$ & * & TNFR1 & $k_{1}$ & $=$ & $5 e-3$ & \\
\hline $2^{2}$ & TRADD & $\rightarrow$ & $\mathrm{CIAP} 1 / 2$ & $k_{2}$ & * & TRADD & $k_{2}$ & $=$ & $2 e-2$ & \multirow{5}{*}{$\begin{array}{l}\text { Formation of Complex } 1 \text { containing TRADD, cIAP1/2, } \\
\text { TRAF2, TRAF5, RIP1 and the TAB/TAK complex }\end{array}$} \\
\hline $3^{3}$ & $\mathrm{CIAP} 1 / 2$ & $\rightarrow$ & TRAF2 & $k_{3}$ & * & $\mathrm{CIAP} 1 / 2$ & $k_{3}$ & $=$ & $1 e-2$ & \\
\hline $4^{4}$ & $\mathrm{CIAP} 1 / 2$ & $\rightarrow$ & TRAF5 & $k_{4}$ & * & $\mathrm{CIAP} 1 / 2$ & $k_{4}$ & $=$ & $8 e-3$ & \\
\hline $5^{5}$ & TRAF2 & $\rightarrow$ & RIP1 & $k_{5}$ & * & TRAF2 & $k_{5}$ & $=$ & $1 e-3$ & \\
\hline $6^{5}$ & TRAF5 & $\rightarrow$ & RIP1 & $k_{6}$ & * & TRAF5 & $k_{6}$ & $=$ & $1 e-3$ & \\
\hline $7^{6}$ & TRADD & $\rightarrow$ & TRAF6 & $k_{7}$ & * & TRADD & $k_{7}$ & $=$ & $2 \mathrm{e}-2$ & Activation of TRAF6 by TRADD \\
\hline $8^{5}$ & TRAF6 & $\rightarrow$ & RIP1 & $k_{8}$ & * & TRAF6 & $k_{8}$ & $=$ & $1 e-4$ & \multirow[t]{2}{*}{ Activation of RIP1 and TAK1 complex by TRAF6 } \\
\hline $9^{7}$ & TRAF6 & $\rightarrow$ & TAK1 complex & $k_{9}$ & * & TRAF6 & $k_{9}$ & $=$ & $1.3 e-4$ & \\
\hline $10^{8}$ & RIP1 & $\rightarrow$ & LUBAC & $k_{10}$ & * & RIP1 & $k_{10}$ & $=$ & $7 e-3$ & \multirow[t]{3}{*}{ Complex 1 ubiquitination by LUBAC and SHARPIN } \\
\hline $11^{9}$ & RIP1 & $\rightarrow$ & SHARPIN & $k_{11}$ & * & RIP1 & $k_{11}$ & $=$ & $7 e-3$ & \\
\hline $12^{7}$ & LUBAC & $\rightarrow$ & TAK1 complex & $k_{12}$ & * & LUBAC & $k_{12}$ & $=$ & $1 e-1$ & \\
\hline 13 & SHARPIN & $\rightarrow$ & IKK complex & $k_{13}$ & * & SHARPIN & $k_{13}$ & $=$ & $1 e-2$ & \multirow[t]{2}{*}{ Activation of IKK complex by Complex 1} \\
\hline 14 & TAK1 complex & $\rightarrow$ & IKK complex & $k_{14}$ & * & TAK1 complex & $k_{14}$ & $=$ & $1 e-1$ & \\
\hline $15^{10}$ & IKK complex & $\rightarrow$ & $\mid \kappa B a$ & $k_{15}$ & * & IKK complex & $k_{15}$ & $=$ & $1 e-2$ & Phosphorylation of IKBa by IKK \\
\hline 16 & $\mathrm{IkBa}$ & $\rightarrow$ & $N F-k B C$ & $k_{16}$ & * & $\mathrm{IkBa}$ & $k_{16}$ & $=$ & $8 e-3$ & Degradation of IkBa forms NF-KB \\
\hline 17 & $N F-k B C$ & $\rightarrow$ & $\mathrm{NF}-\mathrm{kBn}$ & $k_{17}$ & * & $\mathrm{NF}-\mathrm{kBC}$ & $k_{17}$ & $=$ & $1.7 e-2$ & Translocation of NF-KB to nucleus \\
\hline 18 & TAK1 complex & $\rightarrow$ & MKK1/2 & $k_{18}$ & * & TAK1 complex & $k_{18}$ & $=$ & $1.5 e-3$ & \multirow[t]{3}{*}{ Activation of MAP kinases kinases by TAK1 complex } \\
\hline $19^{11}$ & TAK1 complex & $\rightarrow$ & MKK3/6 & $k_{19}$ & * & TAK1 complex & $k_{19}$ & $=$ & $1 e-2$ & \\
\hline 20 & TAK1 complex & $\rightarrow$ & MKK4/7 & $k_{20}$ & * & TAK1 complex & $k_{20}$ & $=$ & $1 e-2$ & \\
\hline 21 & MKK1/2 & $\rightarrow$ & ERK & $k_{21}$ & * & MKK $1 / 2$ & $k_{21}$ & $=$ & $5 e-3$ & \multirow[t]{3}{*}{ Activation of MAP kinases } \\
\hline $22^{12}$ & MKK3/6 & $\rightarrow$ & p38 & $k_{22}$ & * & MKK3/6 & $k_{22}$ & $=$ & $5 e-3$ & \\
\hline 24 & MKK4/7 & $\rightarrow$ & JNK & $k_{23}$ & * & MKK4/7 & $k_{23}$ & $=$ & $5 e-3$ & \\
\hline 24 & p38 & $\rightarrow$ & p38n & $k_{24}$ & * & p38 & $k_{24}$ & $=$ & $5 e-2$ & \multirow[t]{3}{*}{ Translocation of MAP kinases into nucleus } \\
\hline 25 & JNK & $\rightarrow$ & JNKn & $k_{25}$ & * & JNK & $k_{25}$ & $=$ & $5 e-2$ & \\
\hline 26 & ERK & $\rightarrow$ & ERKn & $k_{26}$ & * & ERK & $k_{26}$ & $=$ & $5 e-3$ & \\
\hline 27 & p38n & $\rightarrow$ & AP1 & $k_{27}$ & * & p38n & $k_{27}$ & $=$ & $1 e-2$ & \multirow[t]{3}{*}{ Activation of AP1 by MAP kinases } \\
\hline 28 & ERKn & $\rightarrow$ & AP1 & $k_{28}$ & $*$ & ERKn & $k_{28}$ & $=$ & $1 e-2$ & \\
\hline 29 & JNKn & $\rightarrow$ & AP1 & $k_{29}$ & * & JNKn & $k_{29}$ & $=$ & $1 e-2$ & \\
\hline 30 & AP1 & $\rightarrow$ & Gl promoter & $k_{30}$ & $*$ & AP1 & $k_{30}$ & $=$ & $1 e-1$ & Promoter binding of AP1 and NF-KB for group I genes \\
\hline
\end{tabular}


Table 1 TNFR1 model A (Continued)

\begin{tabular}{|c|c|c|c|c|c|c|c|c|c|c|}
\hline 31 & $\mathrm{NF}-\mathrm{KBn}$ & $\rightarrow$ & Gl promoter & $k_{31}$ & * & $\mathrm{NF}-\mathrm{kBn}$ & $k_{31}$ & $=$ & $5 e-3$ & \multirow{4}{*}{ Group I genes transcription, splicing (1 step) and decay } \\
\hline 32 & G1 promoter & $\rightarrow$ & Gl pre-mRNA & $k_{32}$ & $*$ & Gl promoter & $k_{32}$ & $=$ & $1 e-2$ & \\
\hline 33 & Gl pre-mRNA & $\rightarrow$ & GI mRNA & $k_{33}$ & $*$ & Gl pre-mRNA & $k_{33}$ & $=$ & $5 e-2$ & \\
\hline 34 & Gl mRNA & $\rightarrow$ & Gl mRNA decay & $k_{34}$ & $*$ & Gl mRNA & $k_{34}$ & $=$ & $2 e-3$ & \\
\hline $35^{13}$ & AP1 & $\rightarrow$ & Gll promoter & $k_{35}$ & * & AP1 & $k_{35}$ & $=$ & $1.1 \mathrm{e}-2$ & \multirow[t]{2}{*}{ Promoter binding of AP1 and NF-KB for group II genes } \\
\hline 36 & $\mathrm{NF}-\mathrm{kBn}$ & $\rightarrow$ & Gll promoter & $k_{36}$ & * & $\mathrm{NF}-\mathrm{kBn}$ & $k_{36}$ & $=$ & $4 e-3$ & \\
\hline 37 & Gll promoter & $\rightarrow$ & Gll pre-mRNA/1 & $k_{37}$ & * & Gll promoter & $k_{37}$ & $=$ & $2 e-3$ & \multirow[t]{4}{*}{ Group II genes transcription, splicing (2 steps) and decay } \\
\hline 38 & Gll pre-mRNA1 & $\rightarrow$ & Gll pre-mRNA/2 & $k_{38}$ & $*$ & Gll pre-mRNA/1 & $k_{38}$ & $=$ & $5 e-2$ & \\
\hline 39 & Gll pre-mRNA/2 & $\rightarrow$ & GII mRNA & $k_{39}$ & * & Gll pre-mRNA/2 & $k_{39}$ & $=$ & $5 e-2$ & \\
\hline $40^{13}$ & GIl mRNA & $\rightarrow$ & Gll mRNA decay & $k_{40}$ & * & GII mRNA & $k_{40}$ & $=$ & $1.2 \mathrm{e}-4$ & \\
\hline 41 & AP1 & $\rightarrow$ & Glll promoter & $k_{41}$ & * & AP1 & $k_{41}$ & $=$ & $5 e-3$ & \multirow[t]{2}{*}{ Promoter binding of AP1 and NF-KB for group III genes } \\
\hline 42 & $\mathrm{NF}-\mathrm{kBn}$ & $\rightarrow$ & GIII promoter & $k_{42}$ & $*$ & $\mathrm{NF}-\mathrm{kBn}$ & $k_{42}$ & $=$ & $1 e-4$ & \\
\hline 43 & GIll promoter & $\rightarrow$ & GIII pre-mRNA/1 & $k_{43}$ & $*$ & GIll promoter & $k_{43}$ & $=$ & $1 e-1$ & \multirow[t]{5}{*}{ Group III genes transcription, splicing (3 steps) and decay } \\
\hline 44 & GIII pre-mRNA/1 & $\rightarrow$ & GIII pre-mRNA/2 & $k_{44}$ & * & GIII pre-mRNA/1 & $k_{44}$ & $=$ & $4 \mathrm{e}-4$ & \\
\hline 45 & GIII pre-mRNA/2 & $\rightarrow$ & GIII pre-mRNA/3 & $k_{45}$ & $*$ & GIII pre-mRNA/2 & $k_{45}$ & $=$ & $1 e-3$ & \\
\hline 46 & GIII pre-mRNA/3 & $\rightarrow$ & GIII mRNA & $k_{46}$ & * & GIII pre-mRNA/3 & $k_{46}$ & $=$ & $2 e-4$ & \\
\hline 47 & GIII mRNA & $\rightarrow$ & GIII mRNA decay & $k_{47}$ & * & GIII mRNA & $k_{47}$ & $=$ & $2 e-5$ & \\
\hline 48 & GI $m R N A$ & $\rightarrow$ & $x 1$ & $k_{48}$ & * & Gl mRNA & $k_{48}$ & $=$ & $1 e-5$ & \multirow[t]{5}{*}{ Feedback processes via group I genes or NF-kB } \\
\hline 49 & $N F-K B n$ & $\rightarrow$ & $x 1$ & $k_{49}$ & * & $\mathrm{NF}-\mathrm{kBn}$ & $k_{49}$ & $=$ & $5 e-1$ & \\
\hline 50 & $x 1$ & $\rightarrow$ & $x 2$ & $k_{50}$ & * & $\mathrm{X} 1$ & $k_{50}$ & $=$ & $2 e-3$ & \\
\hline 51 & $x 2$ & $\rightarrow$ & $x 3$ & $k_{51}$ & * & $x 2$ & $k_{51}$ & $=$ & $2 e-3$ & \\
\hline 52 & $x 3$ & $\rightarrow$ & $x 4$ & $k_{52}$ & $*$ & X3 & $k_{52}$ & $=$ & $2 e-3$ & \\
\hline 53 & $x 4$ & $\rightarrow$ & $x 5$ & $k_{53}$ & * & $\mathrm{X} 4$ & $k_{53}$ & $=$ & $2 e-3$ & \multirow{2}{*}{$\begin{array}{l}\text { Steps of the secondary feedback processes } \\
\text { (cytosolic or autocrine signaling): }\end{array}$} \\
\hline 54 & $x 5$ & $\rightarrow$ & $x 6$ & $k_{54}$ & * & $\times 5$ & $k_{54}$ & $=$ & $2 e-3$ & \\
\hline 55 & $x 6$ & $\rightarrow$ & $x 7$ & $k_{55}$ & $*$ & $x 6$ & $k_{55}$ & $=$ & $2 e-3$ & - expression (e.g. translation) \\
\hline 56 & $x 7$ & $\rightarrow$ & $x 8$ & $k_{56}$ & * & $\mathrm{X} 7$ & $k_{56}$ & $=$ & $2 e-3$ & • transport (e.g. secretion) \\
\hline 57 & $x 8$ & $\rightarrow$ & $x 9$ & $k_{57}$ & * & $\mathrm{x} 8$ & $k_{57}$ & $=$ & $2 e-3$ & \multirow[t]{5}{*}{ - signaling (e.g. receptor binding, activation of transcription factors) } \\
\hline 58 & $x 9$ & $\rightarrow$ & $x 10$ & $k_{58}$ & * & X9 & $k_{58}$ & $=$ & $2 \mathrm{e}-3$ & \\
\hline 59 & $x 10$ & $\rightarrow$ & $X 11$ & $k_{59}$ & * & $\times 10$ & $k_{59}$ & $=$ & $2 e-3$ & \\
\hline 60 & $X 11$ & $\rightarrow$ & $X 12$ & $k_{60}$ & * & X11 & $k_{60}$ & $=$ & $2 e-3$ & \\
\hline 61 & $X 12$ & $\rightarrow$ & $X 13$ & $k_{61}$ & $*$ & $X 12$ & $k_{61}$ & $=$ & $2 \mathrm{e}-3$ & \\
\hline
\end{tabular}


Table 1 TNFR1 model A (Continued)

\begin{tabular}{|c|c|c|c|c|c|c|c|c|c|c|}
\hline 62 & $x 13$ & $\rightarrow$ & $x 14$ & $k_{62}$ & * & $\mathrm{x} 13$ & $k_{62}$ & $=$ & $2 e-3$ & \\
\hline 63 & $x 14$ & $\rightarrow$ & IKBa & $k_{63}$ & * & $\mathrm{X} 14$ & $k_{63}$ & $=$ & $2 e-3$ & IKBa feedback activation \\
\hline 64 & $x 14$ & $\rightarrow$ & $Y$ & $k_{64}$ & * & $\mathrm{X} 14$ & $k_{64}$ & $=$ & $1 e-5$ & Group III feedback activation via transcription factor $Y$ \\
\hline 65 & $Y$ & $\rightarrow$ & GIII promoter & $k_{65}$ & * & Y & $k_{65}$ & $=$ & $2 e-3$ & \\
\hline
\end{tabular}

(1-12): in-silico knock-out conditions are performed by setting parameter values $\left(k_{i}\right)$ to 0 for targeted reactions in TRADD KO (1), cIAP1/2 KO (2), TRAF2 KO (3), TRAF5 KO (4) TRAF2/5 DKO (3,4), RIP KO (5), TRAF6 KO (6), TAK1 complex KO (7), LUBAC KO (8), SHARPIN KO (9), IKBa KO (10), MKK3/6 KO (11) and p38 KO (12). (13) Kinetics of Group II mRNA transcription and decay processes were refitted after adding feedback (without feedback: $k_{35}=7 \mathrm{e}-3, k_{40}=1.2 \mathrm{e}-5$ ). Bold italic fonts (reactions $48-65$ ) indicate additional feedback activation pathways required for group III continuous activation. * indicates the multiplication sign. 
enhanced IKB $\alpha$ phosphorylation and $\mathrm{p} 38$ kinase activation due to Signaling Flux Redistribution (Response Rule 5, Additional file 1: Figure S1) [18]. In the remaining conditions, the activation levels of both molecules are very weak (RIP1 KO and TRAF2/5 DKO) or absent (TRADD KO).

It is noteworthy that although there have been previous models on TNF signaling [24,27,28], to our knowledge, this is the first time a single model of TNF signaling with fixed parameter values recapitulates the proinflammatory signaling dynamics in multiple experimental conditions.

To compare our linear response model (TNFR1 model A) simulations with other models that contain more detailed descriptions of IKK [28] and MAPK [29] signaling, using higher order terms and Michaelis-Menten type kinetics, we developed an alternative TNFR1 model B incorporating the relevant reaction details (Additional file 1: Table S3). Notably, the simulations of TNFR1 models A and $B$ show very similar dynamics for a fixed amount of TNF perturbation (Additional file 1: Figure S3). Thus, we concur that our linear response model can be appropriately used for further investigations.

Simulating distinct TNF-induced gene expression patterns Next, we extended the TNFR1 model (we will now simply call TNFR1 model A as TNFR1 model) to simulate downstream proinflammatory gene expression dynamics. Recently, time-series high throughput microarray and quantitative real time PCR experiments on TNF simulated mouse 3T3 fibroblasts cells have revealed 3 distinct groups of upregulated gene expression patterns, with possibly corresponding distinct biological roles [12,30]. The groups were labeled into "early I", "intermediate or middle II" and "late III" response, according to their time to reach peak expressions between $0.5-1,2-3$, and $6-12 \mathrm{~h}$, respectively, after TNF stimulation (Figure 3A) [12,30,31]. Here, we extended the TNFR1 model to simulate the temporal profiles of the 3 groups of gene expressions.

According to our modeling approach, the time to peak activation can be controlled by reaction parameter values and/or the number of signaling intermediates [15,17-20]. Briefly, decreasing (increasing) the activation or transcription parameter value will show lower (sharper) gradients of formation part of the expression profiles. Alternatively, decreasing (increasing) the deactivation or decay parameter value will show lower (sharper) gradients of depletion part of expression profiles (Response Rule 1, Additional file 1: Figure S1). In addition, inserting intermediary reactions between transcription process and gene induction will increase delay for gene expression dynamics (Response rule 2, Additional file 1: Figure S1). The intermediates can represent the complexities of transcription process involving the pre-initiation, initiation, promoter clearance, elongation and termination [32], or post-transcriptional processes such as messenger RNA editing and splicing. Using this approach, the TNFR1 model was extended to simulate the temporal dynamics of groups I, II and III genes. Note that the response rules (Additional file 1: Figure S1) are used to modify an initial signaling topology only after all parameter space has been exhaustively searched, and a reasonable model fit is unable to be achieved [20].

Previous investigations on the 3 groups of genes have indicated distinct mechanisms for the differential dynamical response [12,30]. Hao and Baltimore have found lesser presence of AU Rich Element (ARE) region on the 3'UTR of group III genes, targeted by microRNAs and ARE-binding proteins (such as tristetraprolin) that enhance RNA decay processes. Hence, it was postulated as one possible reason for the lower decay response of group III genes compared with genes from groups I and II [12]. More recently, by studying the kinetics of premRNA and mRNA, Hao and Baltimore observed delays in splicing of groups II and III genes compared to group I genes. The differential delays were suggested as another biological mechanism for the distinct gene profiles [30].

In our extended model, we, therefore, considered both mechanisms to reproduce the temporal profiles of the 3 groups of genes. Notably, our simulations of pre-mRNA and mRNA for all groups of genes matched the data of Hao and Baltimore for the first $60 \mathrm{~min}$ (Additional file 1: Figure S4). However, subsequently for $12 \mathrm{~h}$, although the simulations of groups I and II genes were recapitulated, group III simulation was poor (Figure 3B, blue line). Specifically, reducing the parameter value for the decay term representing lower miRNA and ARE-binding proteins regulating decay processes (Response rule 1, Additional file 1: Figure S1), and adding intermediates (Response rule 2, Additional file 1: Figure S1) to provide delays in RNA splicing in our model were not sufficient to produce the continuous activation of group III genes (Figure 3C, cyan dotted line).

To overcome the shortfall in the model simulations, we hypothesized that novel activation or transcription term(s) (positive feedback) may be present to provide additional flux for the continuous increase in group III expressions (Response rule 4, Additional file 1: Figure S1). This could result from secondary posttranscriptional/translational mechanisms through i) autocrine signaling such as IL-1 [33], IL-6 [34] or TGF- $\beta$ [35] signaling (Figure 3D), or ii) cytosolic feedback mechanisms specifically for group III genes [36] (Figure 3E). Thus, a novel feedback mechanism predominantly affecting the transcription of group III genes was added to the TNFR1 model (Table 1, equations 30-65 and Additional file 2).

The modified TNFR1 model with feedback mechanisms to group III genes produced simulations that matched all 3 groups of gene expression profiles 


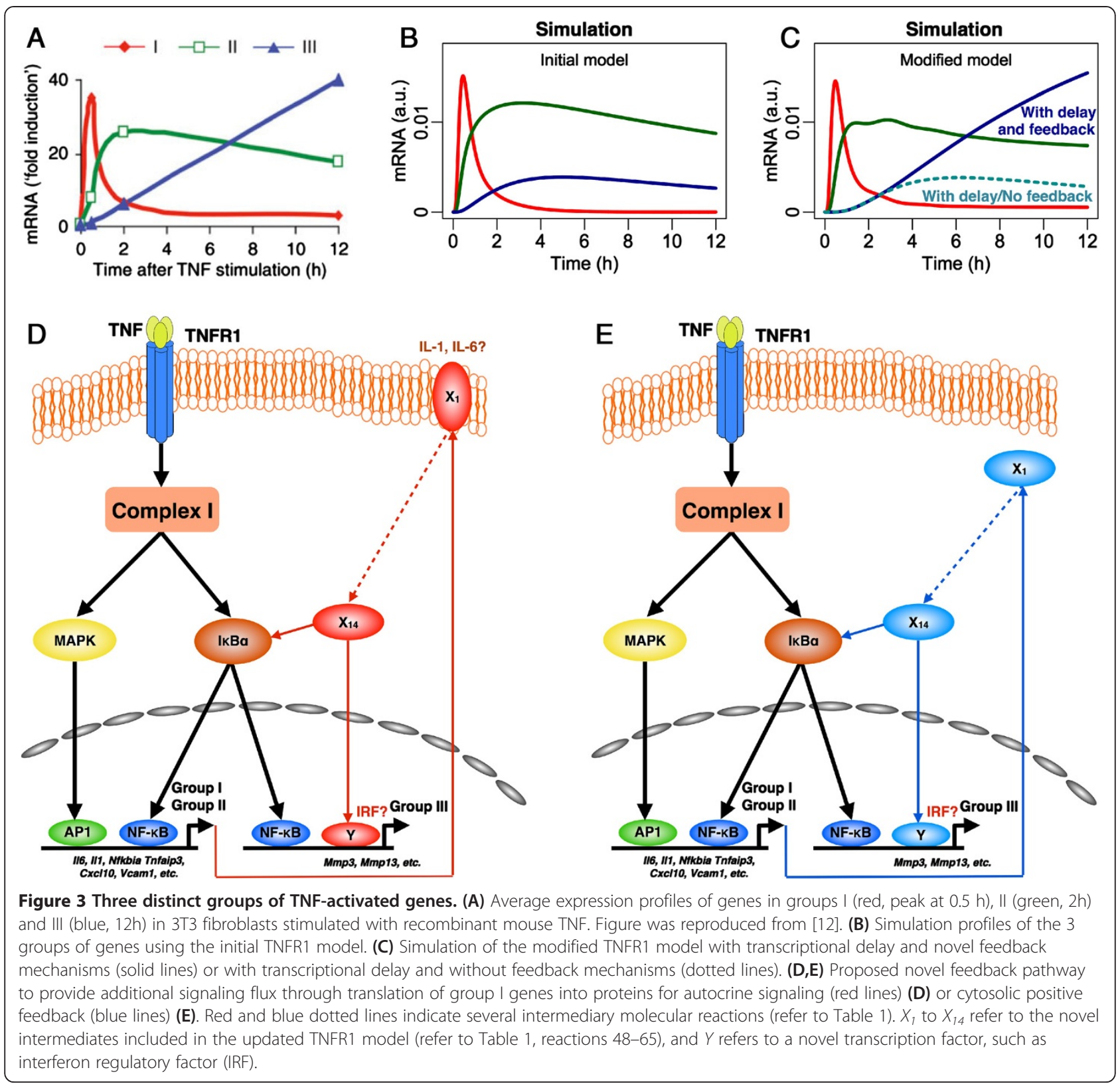

(Figure 3A and C, solid lines). To scrutinize the feedback mechanism, we re-monitored the simulation profile of NF- $\mathrm{kB}$ for 6 hours (Additional file 1: Figure S5). The resultant profile mimics the damped oscillatory dynamics of NF- $\mathrm{kB}$ previously observed in murine fibroblasts [36]. Overall, these data suggest that low miRNA regulation and additional delay in RNA splicing are not sufficient to produce the continuous activation of group III genes, and that a novel transcription process, possibly through secondary posttranscriptional/translational autocrine signaling, such as IL-1 signaling or other novel feedback mechanisms that activate NF-kB, and not MAPK (Additional file 1: Figure S5), are required.

\section{Predicting key target for regulating proinflammatory} response

Now that the TNFR1 model is able to successfully simulate the three groups of upregulated genes in wildtype, we investigated the significance and effect of removing or suppressing key intracellular signaling molecules for controlling proinflammatory response, in silico.

It is well known that TNFR1 signaling is enhanced in proinflammatory diseases and cancer [1-4]. To investigate which known molecules would be potential target to regulate the cell survival or proinflammatory activity, we performed in silico KOs of all possible signaling molecules within the TNFR1 model. In total, we simulated groups I, II and III dynamic gene expressions in 12 
(TRADD, cIAP1/2, TRAF2, TRAF5, TRAF6, RIP1, SHARPIN, LUBAC, TAK1 complex (TAK1/TAB1/2), IкB $\alpha, M K K 3 / 6$ and p38) KO conditions and compared with wildtype profiles (Additional file 1: Figure S6).

Among the candidates, the removal of TAK1 complex or RIP1 produced the most noticeable downregulation of all 3 gene groups, which chiefly consist of well-known proinflammatory mediators (Figure 4). However, in TAK1 complex KO, our simulations show almost no induction for group 1 genes. The substantial impairment

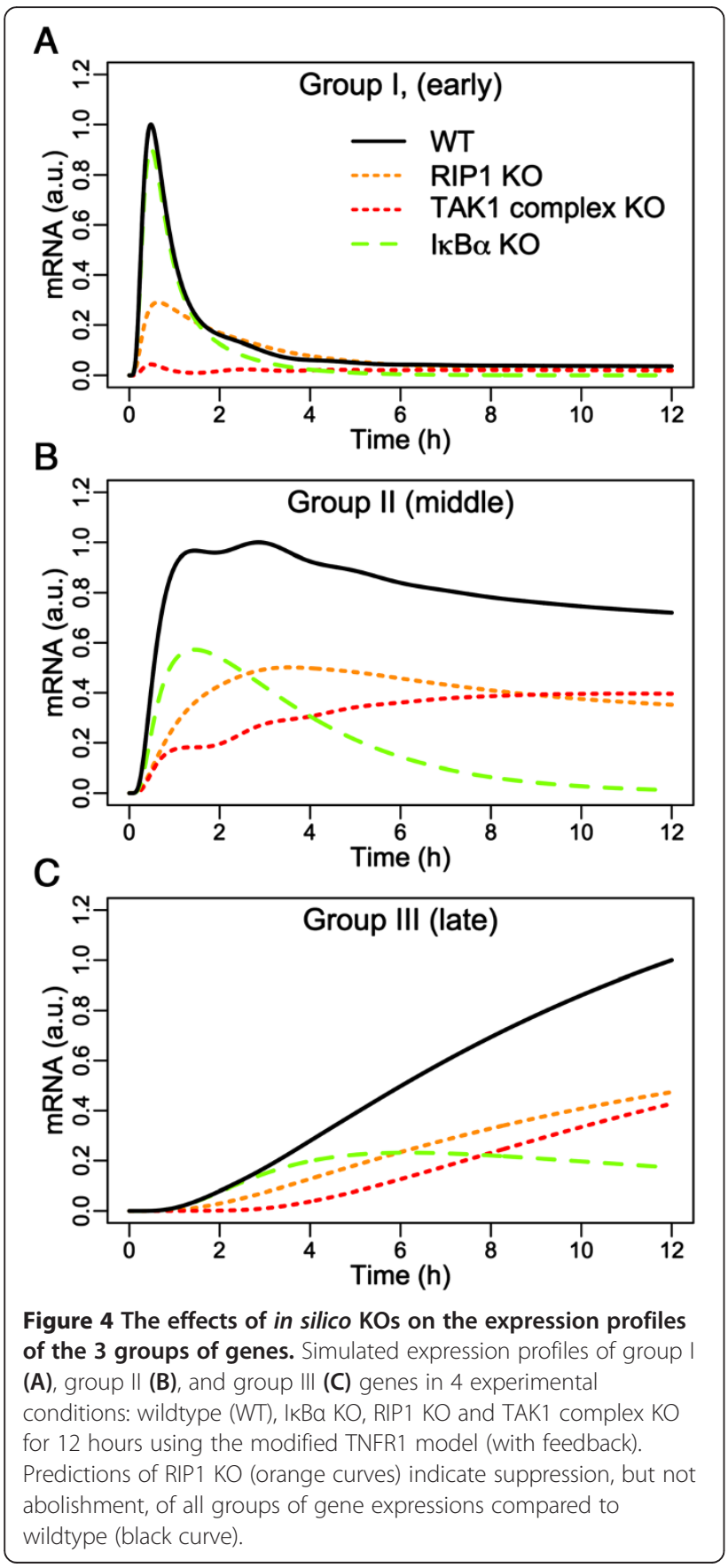

in gene expressions (> 90\%) is usually detrimental to the general survivability of living cells, and this has been particularly demonstrated in TAK1-deficient mice [37,38]. RIP1, on the other hand, showed about $50-70 \%$ impairment compared to wildtype peak expressions. Our simulations, therefore, suggest that RIP1 is possibly a crucial single molecule target for controlling enhanced proinflammatory response due to TNFR1 signaling in proinflammatory disease conditions, such as in rheumatoid arthritis, without compromising the normal functioning of other cellular activities.

\section{Experimental inhibition of RIP1 downregulates proinflammatory genes in TNF stimulation}

To verify the predictions of TNFR1 model simulations, we prepared corresponding MEF and BALB/3T3 cells treated with TNF in wildtype and in RIP1 suppression. Necrostatin-1 (Nec-1) was originally identified as a potent small molecule inhibitor of necroptosis or nonapoptotic cell death [39]. Further interests in Nec-1 led to its specificity towards the inhibition of RIP1 [40]. Although Nec-1 has recently been extensively studied, its effect on the expressions of groups I, II and III genes in TNF stimulation remains largely unknown. Therefore, here, we used Nec-1 to suppress RIP1 in vivo.

To check the effect of cell death by Nec-1, we compared MEF and BALB/3T3 cells treated with different doses of Nec-1 in the presence or absence of TNF (Additional file 1: Figure S7). The data revealed that Nec-1 has no substantial effect on cell death after $24 \mathrm{~h}$ incubation, and hence, could be tested for its efficacy on the 3 groups of genes. We next performed quantitative RT-PCR for a total of 10 genes: Il6, Tnfaip3, Jun, Nfkbia (group I), Ccl7, Vcam1, Cxcl10 (group II), and Mmp3, Mmp13, Enpp2 (group III). We intentionally included key proinflammatory mediators, genes of matrix metalloproteinase ( $\mathrm{Mmp3}$, Mmp13), which are known to degrade collagen in cartilage and thereby enhance rheumatoid arthritis and osteoarthritis progression [41-44].

A previous study has shown that $30 \mu \mathrm{M}$ of Nec-1 effectively inhibited RIP1 kinase activity [41]. Therefore, we investigated gene expressions for cells stimulated with $10 \mathrm{ng} / \mathrm{mL}$ TNF, in the presence or absence of $30 \mu \mathrm{M} \mathrm{Nec-}$ 1 for a period of 10 hours with measurements made at least every hour (Figure 5). Remarkably, as predicted by the TNFR1 model, RIP1 inhibition by Nec-1 resulted in the suppression of all 3 groups of genes. The effect of suppressing RIP1 is significant for groups I and II genes in both MEF and BALB/3T3 cells, especially during the first 2-3 hours after stimulation. For group III genes, Nec-1 had more pronounced effect in MEF compared with BALB/3T3 cells. Overall, these results are consistent with the TNFR1 model predictions that suppressing RIP1 in 


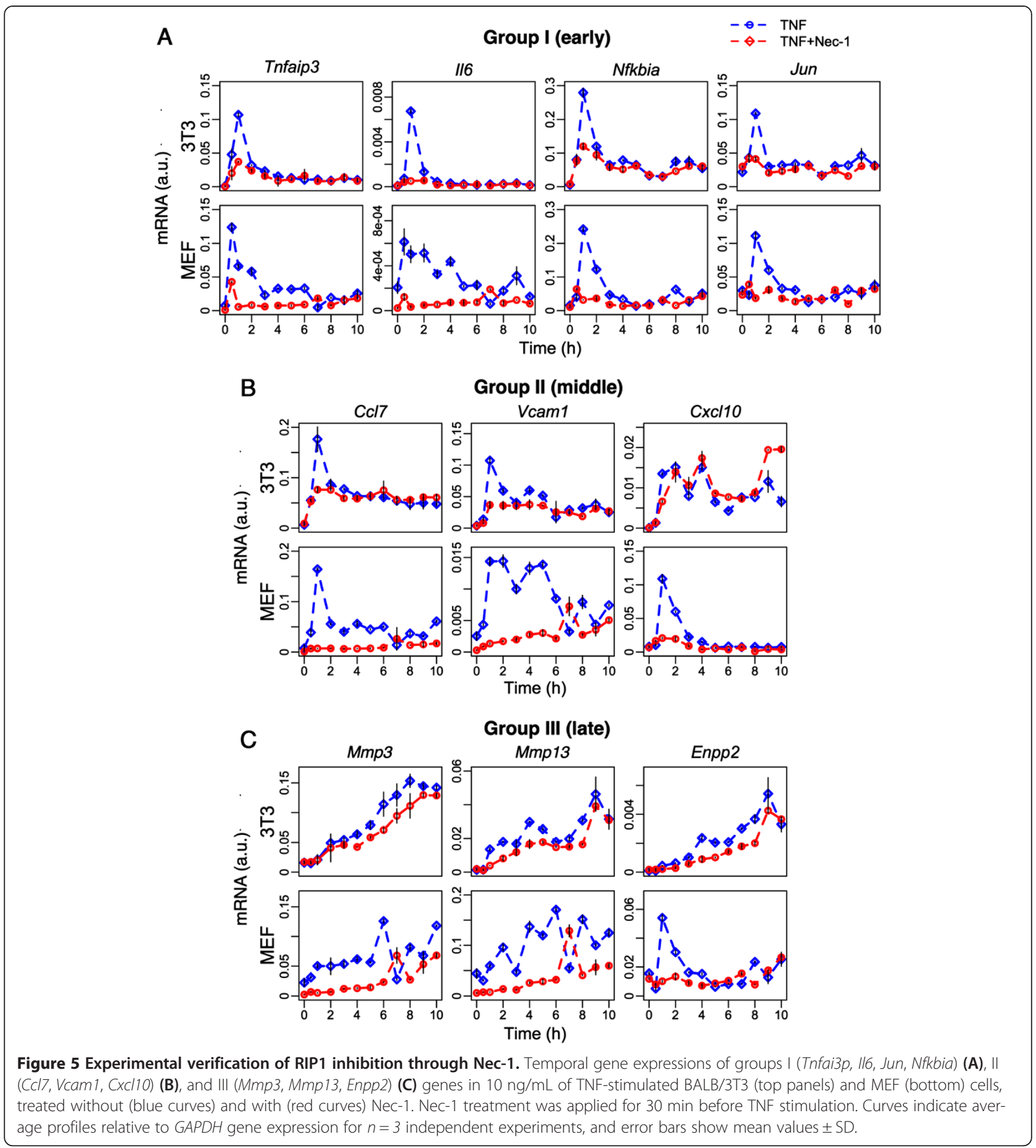

TNF stimulation significantly impairs the activation of all 3 groups of genes.

\section{Discussion}

TNF is a crucial cytokine that regulates myriad vital cellular processes. However, its levels are enhanced in major proinflammatory diseases. Here, to understand the TNF-induced proinflammatory signaling process, and to carefully regulate its dynamic response, a systems biology approach was adopted. We first developed a dynamic computational model using well-established publicly available experimental data of NF-kB, MAP kinase p38, and the average profiles of 3 groups of 180 upregulated genes in mouse fibroblast cells.

Despite the simplicity of using first-order response equations to simulate the profiles of the intracellular molecules, 
the computational model of TNFR1 recapitulated the experimental response in wildtype and several mutant conditions for NF-kB and $\mathrm{p} 38$ activations. This result is surprising, as we know that the innate immune response of TNF is highly complex. It is important to note here that there have been previous other computational efforts on NF- $\mathrm{kB}$ and MAPK signaling that had utilized detailed biochemical reactions modeling, to elucidate local properties of signal transduction, such as the ability of common molecules to produce distinct feedback mechanisms to different stimuli $[24,45,46]$. In our work, however, we have shown that even a simpler representation of the signal transduction pathways, through first order response equations and the law of mass conservation can reproduce experimental dynamics. This strongly indicates the presence of simple organizing rules governing the deterministic population average signaling response [47-52].

Next, through the analyses of downstream temporal gene expression profiles, the model suggests the presence of additional novel post-transcriptional/translational processes that is required for the continuous activation of group III genes. This result is additional to previous postulations, which had indicated that the continuous activation is due to lesser ARE region for group III genes leading to a very low decay process [12], and due to the presence of differential delays in the RNA splicing process [30]. Our model suggests that, on top of these effects, a novel timedelayed secondary transcriptional mechanism is required.

Literature survey indicates that the novel positive feedback processes could be a result of autocrine signaling, example through IL-1 or IL-6, or derive from a still unknown intracellular feedback mechanisms regulating mainly the promoter regions of group III genes. For example, the role of interferon regulatory factor (IRF) family in inducing $\mathrm{Ccl} 5$ or RANTES expression, which belongs to one of the group 3 genes, is reported in a previous study [53], however, was not considered in the initial TNFR1 model. It is, therefore, necessary to perform further experimental work to confirm and elucidate the exact mechanisms for the continuous activations of group III genes.

On the other hand, for down-regulating TNF signaling, which is enhanced in several proinflammatory diseases and cancer, we performed the simulations for $12 \mathrm{in}$ silico KOs of signaling molecules. The resultant simulations indicated that RIP1 is a major regulator of the 3 groups of upregulated gene expressions. To verify the result, we performed experiments on MEF and BALB/3T3 cells using Nec-1 as an inhibitor of RIP1. The measurement of 10 genes belonging to groups I (Il6, Tnfaip3, Jun, Nfkbia), II (Ccl7, Vcam1, Cxcl10) and III (Mmp3, Mmp13, Enpp2) all showed significant impairment with Nec-1 compared to wildtype.

Most importantly, the expressions of key proinflammatory genes such as Il6, Vcam1, Ccl7, Mmp3, Mmp13, enhanced in rheumatoid arthritis and osteoarthritis $[41,44]$, were reduced. In particular are the levels of matrix metalloproteinase genes $M m p 3, M m p 13$, which are known to directly affect type II collagen in bone cartilages and degrade the extracellular matrix. Although recent therapeutics have been focusing on the specific regulations of MMPs [42-44,54], it remains to be seen what effect such treatments will have on other proinflammatory or vital genes.

In summary, our approach provides a systemic analysis of TNFR1 signaling, and suggests Nec-1 is potentially an important therapeutic target for effectively regulating major proinflammatory mediators in chronic diseases where TNF is overexpressed.

\section{Materials and methods Computational model}

The model is based on perturbation-response approach [15,17-20]. The basic principle behind the approach is to induce a controlled perturbation of input reaction species of a system (TNFR1), and monitor the response of the activation/concentration levels of other output species (e.g. TAK1, p38, NF-кB, Il6, etc.) from steady-state. To briefly explain the principle, let a stable network consisting of $n$ species be perturbed from the reference steady-state. In general, the resultant changes in the concentration of species are governed by the kinetic evolution equation $[13,14]$ :

$$
\frac{\partial X_{i}}{\partial t}=F_{i}\left(X_{1}, X_{2}, . ., X_{n}\right), \quad i=1, . ., n
$$

where the corresponding vector form of equation 1 is $\frac{\partial \boldsymbol{X}}{\partial t}=\boldsymbol{F}(\boldsymbol{X}) . \boldsymbol{F}$ is a vector of any non-linear function including diffusion and reaction of the species vector $X=$ $\left(X_{1}, X_{2}, . ., X_{n}\right)$, which represents activated concentration levels of reaction species. The response to perturbation can be written by $X=X_{0}+\delta X$, where $X_{0}$ is the reference steady-state vector and $\delta \boldsymbol{X}$ is the relative response from steady-states $\left(\delta \boldsymbol{X}_{t=0}=\mathbf{0}\right)$.

The generally non-linear kinetic evolution equation 1 can be approximated or linearized by using Taylor series:

$$
\frac{\partial \delta \boldsymbol{X}}{\partial t}=\frac{\partial \boldsymbol{F}(\boldsymbol{X})}{\partial \boldsymbol{X}} \delta \boldsymbol{X}+\frac{\partial^{2} \boldsymbol{F}(\boldsymbol{X})}{\partial \boldsymbol{X}^{2}} \delta \boldsymbol{X}^{2}+\ldots
$$

As the general volume of perturbing substance is usually very small (order of $1 \%$ ) compared to the total volume of cells that are perturbed [55], now consider a small perturbation around the steady-state in equation 2, in which higher-order terms become negligible and result in the approximation of the first-order term. In vector form $\left.\frac{\partial \delta X}{d t} \cong \frac{\partial F(X)}{\partial X}\right|_{X=X_{0}} \delta X$ (note the change from partial derivative to total derivative of time), where the zeroth order term $\boldsymbol{F}\left(\boldsymbol{X}_{0}\right)=0$ at the steady-state $\boldsymbol{X}_{0}$ 
and the Jacobian matrix, or linear stability matrix, is $\boldsymbol{J}=\left.\frac{\partial \boldsymbol{F}(\boldsymbol{X})}{\partial \boldsymbol{X}}\right|_{X=X_{0}}$. The elements of $\boldsymbol{J}$, based on the initial activation topology, are chosen by fitting $\delta X$ with corresponding experimental profiles. Hence, the amount of response (flux propagated) along a biological pathway can be approximated using first order mass-action response, i.e. $\frac{d \delta \boldsymbol{X}}{d t}=J \delta \boldsymbol{X}$. That is, the basic principle so far suggests that the response rate of species in a massconserved system at an initial steady-state can be approximated by first order mass-action response equations, given a small perturbation to one or more species.

Note that Jacobian matrix elements (or response coefficients) can include not only reaction information, but also spatial information such as diffusion and transport mechanisms. Thus, each species in the perturbationresponse model can represent a molecule, a different modified state of a molecule (e.g. ubiquitinated state) or a molecular process such as diffusion, endocytosis, etc. That is, each species in the biological network does not necessarily represent a specific molecular species. For illustration, in a pathway $X_{1} \rightarrow X_{2} \rightarrow X_{3} \rightarrow X_{4} \rightarrow X_{5}, X_{1}$ to $X_{5}$ can each be a different species or the same species at different stages in signaling, for example, $X_{1}$ being internalized (becoming $X_{2}$ ), transported to a different organelle $\left(X_{3}\right)$, ubiquitinated $\left(X_{4}\right)$ and become part of a protein complex $\left(X_{5}\right)$.

The complete SBML version of TNFR1 Models A \& B are available in Additional file 2.

\section{Sensitivity analysis}

We performed a sensitivity analysis to test the robustness of the optimized model parameters using the COPASI sensitivities module with default values. The variation in the response of signaling molecules/steps, $x_{i}$ $(t)$, was analyzed when a small variation of each model parameter $k_{j}$ was applied. The response sensitivity coefficient [56] of the $i^{\text {th }}$ molecule with regard to the $j^{\text {th }}$ parameter is defined by

$$
R_{i, j}=\frac{\partial x_{i}(t)}{\partial k_{j}} \frac{k_{j}}{x_{i}(t)}
$$

The obtained values, $R_{i, j}$ are then scaled, to reflect the relative changes in response, such as a change of $p \%$ in the value of parameter $k_{j}$, results in a $R_{i j} \cdot p \%$ change in the value of the peak activation of the $i^{\text {th }}$ molecule. The response sensitivity coefficients of $\mathrm{p} 38$, IK $\mathrm{B} \alpha$, and groups I, II and III genes were obtained at peak time $\left(t=15 \mathrm{~min}\right.$ for $\mathrm{p} 38$ and $\mathrm{I}_{\kappa} \mathrm{B}, 30 \mathrm{~min}, 2 \mathrm{~h}$ and $12 \mathrm{~h}$ for groups I, II and III respectively, see Additional file 1: Table S2).

\section{Experiments}

\section{Reagents and cell culture}

Recombinant mouse TNF was purchased from R\&D systems. Necrostatin-1 was purchased from Merck Millipore. 3T3 cells were obtained from JCRB cell bank. 3T3 and MEF were grown in DMEM (Nissui Seiyaku Co.) containing $10 \%$ calf serum, $100 \mathrm{U} / \mathrm{mL}$ of penicillin at $37^{\circ} \mathrm{C}$ in a $5 \% \mathrm{CO}_{2}$ humidified atmosphere.

\section{Evaluation of cell survival by 3-(4,5-dimethylthiazol-2-yl)- 2,5-diphenyltetrazolium bromide (MTT) assay}

The sensitivity of cells to hyperosmotic stress was measured with the MTT colorimetric assay in 96-well plates. Cells $\left(2 \times 10^{4}\right)$ were inoculated in each well and incubated for $24 \mathrm{~h}$. Thereafter, $50 \mu \mathrm{L}$ of MTT ( $2 \mathrm{mg} / \mathrm{mL}$ in PBS) was added to each well and the plates were incubated for a further $2 \mathrm{~h}$. The resultant formazan was dissolved with $100 \mu \mathrm{L}$ of dimethyl sulfoxide after aspiration of culture medium. Plates were placed on a plate shaker for $1 \mathrm{~min}$ and then read immediately at $570 \mathrm{~nm}$ using TECAN microplate reader with Magellan software (Männedorf, Switzerland).

\section{Real-time PCR analysis}

Total cellular RNA was extracted from cells using the TRIzol reagent according to the manufacturer's instructions (Invitrogen). One microgram of RNA was reversetranscribed using a first-strand cDNA synthesis kit (ReverTra Ace $;$; Toyobo). Quantitative real-time PCR was performed using SYBR premix Ex Taq (Takara) on the Applied Biosystems StepOnePlus ${ }^{\mathrm{TM}}$ according to the technical brochure of the company. RT-PCR primers designed in this study are listed in Additional file 1: Table S4. Quantitative measurements were determined using the $\Delta \Delta \mathrm{Ct}$ method and expression of GAPDH was used as the internal control. Melt curve analyses of all real-time PCR products were performed and shown to produce the sole DNA duplex.

\section{Additional files}

Additional file 1: Figure S1. Response rules. Figure S2. Experimental raw data used for model fitting. Figure S3. Experimental vs. simulated profiles of $\mathrm{IKBa}$ and $\mathrm{p} 38$ activations in wildtype and mutant conditions using TNFR1 model B. Figure S4. Simulation of pre-mRNA and mRNA expression profiles of groups I, II and III genes. Figure S5. Simulation of NF$\mathrm{KB}$ activation profiles with and without feedback mechanisms. Figure S6. The effects of in silico KOs on the expression profiles of groups I, II and III genes. Table S1. Estimation of the relative intensities of $\mid \mathrm{KBa}$ and p38 activation dynamics. Table S2. Sensitivity analysis of TNFR1 model A. Table S3. TNFR1 model B details. Table S4. List of primer sequences for RT-PCR, Additional file 2: TNFR1 models A \& B in SBML format.

\section{Abbreviations}

TNF: Tumor necrosis factor; MAP: Mitogen-activated protein; NF-kB: Nuclear factor-kB; MEF: Murine embryonic fibroblast; KO: Knock out; RIP1: Receptorinteracting protein 1; Nec-1: Necrostatin-1; TNFR: TNF receptor; DD: Death 
domain; TNFRSF: TNFR superfamily; TRADD: Tumor necrosis factor receptor 1 associated death domain protein; CIAP: Cellular inhibitor of apoptosis proteins; LUBAC: Linear ubiquitin chain assembly complex; HOIL-1: Hemeoxidized iron regulatory protein 2 ubiquitin ligase-1; HOIP: HOIL-1-interacting protein; SHARPIN: SH3 and multiple ankyrin repeat domains proteinassociated RH domain interacting protein; TAK1: Transforming growth factor $\beta(T G F \beta)$-activated kinase 1; AP: Activating protein; FADD: fas-associated death domain protein; TRAlL: TNF-related apoptosis-inducing ligand; DKO: Double knock out; mRNA: Messenger RNA; JNKm: c-Jun N-terminal kinases; ERK: Extracellular signal-regulated kinase; IKB: Inhibitors of NF-KB; ARE: AU rich element; miRNA: micro RNA; IL: Interleukin; RT-PCR: Reverse transcription polymerase chain reaction; IRF: Interferon regulatory factor; MMP: Matrix metalloproteinase.

\section{Competing interests}

The authors declare that they have no competing interests.

\section{Authors' contributions}

$\mathrm{KH}$ and $\mathrm{KS}$ conceptualized and designed the study. $\mathrm{KH}$ and ST performed the wet experiments. VP and KS performed the computational simulations. MT provided cells, reagents and discussions. KH and KS wrote the paper. All authors read and approved the final manuscript.

\section{Acknowledgements}

We thank Kiyotoshi Sato, Tomoyoshi Soga and Mitsuhiro Kitagawa for experimental support.

\section{Funding}

This work was supported by the Japan Society for the Promotion of Science (JSPS) Grants-in-Aid for Scientific Research J13108 (K.S.), Scientific Research F13804 (K.H.), and Tsuruoka City, Yamagata Prefecture.

Received: 28 August 2013 Accepted: 1 November 2013

Published: 7 November 2013

\section{References}

1. O'Malley WE, Achinstein B, Shear MJ: Action of bacterial polysaccharide on tumours: II: damage of sarcoma 37 by serum of mice treated with serratia marcescens polysaccharide, and induced tolerance. J Natl Cancer Inst 1962, 29:1169-1175.

2. Balkwill F: Tumour necrosis factor and cancer. Nat Rev Cancer 2009, 9:361-371.

3. Locksley RM, Killeen N, Lenardo MJ: The TNF and TNF receptor superfamilies: integrating mammalian biology. Cell 2001, 104:487-501.

4. Bradley JR: TNF-mediated inflammatory disease. J Patho/ 2008, 214:149-160.

5. van Horssen R, Ten Hagen TL, Eggermont AM: TNF-alpha in cancer treatment: molecular insights, antitumor effects, and clinical utility. Oncologist 2006, 4:397-408

6. Falschlehner C, Boutros M: Innate immunity: regulation of caspases by IAP-dependent ubiquitylation. EMBO J 2012, 31:2750-2752.

7. Tada K, Okazaki T, Sakon S, Kobarai T, Kurosawa K, Yamaoka S, Hashimoto H, Mak TW, Yagita H, Okumura K, Yeh WC, Nakano H: Critical roles of TRAF2 and TRAF5 in tumor necrosis factor-induced NF-KB activation protection from cell death. J Biol Chem 2001, 276:36530-36534.

8. Funakoshi-Tago M, Kamada N, Shimizu T, Hashiguchi Y, Tago K, Sonoda Y, Kasahara T: TRAF6 negatively regulates TNFalpha-induced NF-kappaB activation. Cytokine 2009, 45:72-79.

9. Fallahi-Sichani M, Flynn JL, Linderman JJ, Kirschner DE: Differential risk of tuberculosis reactivation among anti-TNF therapies is due to drug binding kinetics and permeability. J Immunol 2012, 188:3169-3178.

10. Wiens A, Venson R, Correr CJ, Otuki MF, Pontarolo R: Meta-analysis of the efficacy and safety of Adalimumab, Etanercept, and Infliximab for the treatment of rheumatoid arthritis. Pharmacotherapy 2010, 30:339-353.

11. Ermolaeva MA, Michallet MC, Papadopoulou N, Utermöhlen O, Kranidioti $K$, Kollias G, Tschopp J, Pasparakis M: Function of TRADD in tumor necrosis factor receptor 1 signaling and in TRIF-dependent inflammatory responses. Nat Immunol 2008, 9:1037-1046.

12. Hao S, Baltimore D: The stability of mRNA influences the temporal order of the induction of genes encoding inflammatory molecules. Nat Immunol 2009, 10:281-288.
13. Ross J: Determination of complex reaction mechanisms: analysis of chemical, biological and genetic networks. J Phys Chem A 2008, 112:2134-2143.

14. Moran F, Vlad MO, Bustos M, Trivino JC, Ross J: Species connectivities and reaction mechanisms from neutral response experiments. J Phys Chem $A$ 2007, 111:1844-1851.

15. Selvarajoo K, Tomita M, Tsuchiya M: Can complex cellular processes be governed by simple linear rules? J Bioinform Comput Biol 2009, 7:243-268.

16. Vance W, Arkin A, Ross J: Determination of causal connectivities of species in reaction networks. Proc Natl Acad Sci USA 2002, 99:5816-5821.

17. Selvarajoo K: Discovering differential activation machinery of the toll-like receptor 4 signaling pathways in MyD88 knockouts. FEBS Lett 2006, 580:1457-1464.

18. Selvarajoo K, Takada Y, Gohda J, Helmy M, Akira S, Tomita M, Tsuchiya M, Inoue J, Matsuo K: Signaling flux redistribution at toll-like receptor pathway junctions. PLOS ONE 2008, 3:e3430. DOI:10.1371/journal. pone.0003430.

19. Helmy M, Gohda J, Inoue J, Tomita M, Tsuchiya M, Selvarajoo K: Predicting novel features of Toll-like receptor 3 signaling in macrophages. PLOS ONE 2009, 4:e4661. DOl: 10.1371/journal.pone.0004661.

20. Piras V, Hayashi K, Tomita M, Selvarajoo K: Enhancing apoptosis in TRAIL-resistant cancer cells using fundamental response rules. Sci Rep 2011, 1:144. DOI:10.1038/srep00144.

21. Devin A, Cook A, Lin Y, Rodriguez Y, Kelliher M, Liu Z: The distinct roles of TRAF2 and RIP in IKK activation by TNF-R1: TRAF2 recruits IKK to TNF-R1 while RIP mediates IKK activation. Immunity 2000, 12:419-429.

22. Wertz IE, Dixit VM: Regulation of death receptor signaling by the ubiquitin system. Cell Death Differ 2010, 17:14-24.

23. Hoops S, Sahle S, Gauges R, Lee C, Pahle J, Simus N, Singhal M, Xu L, Mendes P, Kummer U: COPASI-a COmplex PAthway SImulator. Bioinformatics 2006, 22:3067-3074.

24. Werner SL, Barken D, Hoffmann A: Stimulus specificity of gene expression programs determined by temporal control of IKK activity. Science 2005, 309:1857-1861.

25. Tasseff R, Nayak S, Salim S, Kaushik P, Rizvi N, Varner JD: Analysis of the molecular networks in androgen dependent and independent prostate cancer revealed fragile and robust subsystems. PLoS One 2010, 5:e8864. DOI:10.1371/journal.pone.0008864

26. Carroll DA: Chemical laser modelling with genetic algorithms. AIAA 1996, 34:338-346

27. Werner SL, Kearns JD, Zadorozhnaya V, Lynch C, O'Dea E, Boldin MP, Ma A, Baltimore D, Hoffmann A: Encoding NF-kB temporal control in response to TNF: distinct roles for the negative regulators IKBa and A20. Genes Dev 2008, 22:2093-2101.

28. Cho KH, Shin SY, Lee HW, Wolkenhauer O: Investigations into the analysis and modeling of the TNF alpha-mediated NF-kappa B-signaling pathway. Genome Res 2003, 13:2413-2422.

29. Kholodenko BN: Negative feedback and ultrasensitivity can bring about oscillations in the mitogen-activated protein kinase cascades. Eur J Biochem 2000, 267:1583-1588.

30. Hao S, Baltimore D: RNA splicing regulates the temporal order of TNF-induced gene expression. Proc Natl Acad Sci U S A 2013, 110:11934-11939.

31. Tian B, Nowak DE, Brasier AR: A TNF-induced gene expression program under oscillatory NF-kappaB control. BMC Genomics 2005, 6:137-154.

32. Solomon EP, Berg LR, Martin DW: Biology. 9th edition. Thomson Brooks/ Cole: Pacific Grove; 2011

33. Chaudhry SI, Hooper S, Nye E, Williamson P, Harrington K, Sahai E: Autocrine IL-1beta-TRAF6 signalling promotes squamous cell carcinoma invasion through paracrine TNFalpha signalling to carcinoma-associated fibroblasts. Oncogene 2013, 32:747-758.

34. Grivennikov S, Karin M: Autocrine IL-6 signaling: a key event in tumorigenesis? Cancer Cell 2008, 13:7-9.

35. Ihn H: Autocrine TGF-beta signaling in the pathogenesis of systemic sclerosis. J Dermatol Sci 2008, 49:103-113.

36. Hoffmann A, Levchenko A, Scott ML, Baltimore D: The IKB-NF-kB signaling module: temporal control and selective gene activation. Science 2002, 298:1241-1245.

37. Tang M, Wei X, Guo Y, Breslin P, Zhang S, Zhang S, Wei W, Xia Z, Diaz M, Akira S, Zhang J: TAK1 is required for the survival of hematopoietic cells and hepatocytes in mice. J Exp Med 2008, 205:1611-1619. 
38. Lamothe B, Lai Y, Xie M, Schneider MD, Darnay BG: TAK1 is essential for osteoclast differentiation and is an important modulator of cell death by apoptosis and necroptosis. Mol Cell Biol 2013, 33:582-595.

39. Degterev A, Huang Z, Boyce M: Chemical inhibitor of nonapoptotic cell death with therapeutic potential for ischemic brain injury. Nat Chem Biol 2005, 1:112-119.

40. Degterev A, Hitomi J, Germscheid M, Ch'en IL, Korkina O, Teng X, Abbott D, Cuny GD, Yuan C, Wagner G, Hedrick SM, Gerber SA, Lugovskoy A, Yuan J: Identification of RIP1 kinase as a specific cellular target of necrostatins. Nat Chem Biol 2008, 4:313-321.

41. Liacini A, Sylvester J, Li WQ, Zafarulla M: Inhibition of interleukin-1stimulated MAP kinases, activating protein-1 (AP-1) and nuclear factor kappa B (NF-kappa B) transcription factors down-regulates matrix metalloproteinase gene expression in articular chondrocytes. Matrix Biol 2002, 21:251-262.

42. Liacini A, Sylvester J, Li WQ, Huang W, Dehnade F, Ahmad M, Zafarullah M: Induction of matrix metalloproteinase-13 gene expression by TNF-alpha is mediated by MAP kinases, AP-1, and NF-kappaB transcription factors in articular chondrocytes. Exp Cell Res 2003, 288:208-217.

43. Sellam J, Berenbaum F: The role of synovitis in pathophysiology and clinical symptoms of osteoarthritis. Nat Rev Rheumatol 2010, 6:625-635.

44. Roman-Blas JA, Jimenez MD: NF-KB as a potential therapeutic target in osteoarthritis. Osteoarthritis Cartilage 2006, 14:839-848.

45. Santos SD, Verveer PJ, Bastiaens PI: Growth factor-induced MAPK network topology shapes Erk response determining PC-12 cell fate. Nat Cell Biol 2007, 9:324-330

46. Bruggeman FJ, Westerhoff HV, Hoek JB, Kholodenko BN: Modular response analysis of cellular regulatory networks. J Theor Biol 2002, 218:507-520.

47. Selvarajoo K, Tomita M: Physical laws shape biology. Science 2013, 339:646.

48. Selvarajoo K: Interpreting the dynamics and patterns of living systems. Bioscience 2013, 63:721-722.

49. Selvarajoo K: Macroscopic law of conservation revealed in the population dynamics of Toll- like receptor signaling. Cell Commun Signal 2011, 9:9.

50. Selvarajoo $\mathrm{K}$ : Understanding multimodal biological decisions from single cell and population dynamics. WIREs Syst Biol Med 2012, 4:385-399.

51. Selvarajoo K: Uncertainty and certainty in cellular dynamics. Front Genet 2013, 4:68. DOl:10.3389/fgene.2013.00068.

52. Selvarajoo K, Giuliani A: Finding self-organization from the dynamic gene expressions of innate immune responses. Front Physiol 2012, 3:192. DOl:10.3389/fphys.2012.00192.

53. Yarilina A, Park-Min KH, Antoniv T, Hu X, Ivashkiv LB: TNF activates an IRF1- dependent autocrine loop leading to sustained expression of chemokines and STAT1-dependent type I interferon-response genes. Nat Immunol 2008, 9:378-387.

54. Kaneva MK, Kerrigan MJ, Grieco P, Curley GP, Locke IC, Getting SJ: Chondroprotective and anti-inflammatory role of melanocortin peptides in TNF-alpha activated human C-20/A4 chondrocytes. Br J Pharmacol 2012, 167:167-179.

55. Theobald U, Mailinger W, Baltes M, Rizzi M, Reuss M: In vivo analysis of metabolic dynamics in Saccharomyces cerevisiae: I: Experimental observations. Biotechnol Bioeng 1997, 55:305-316.

56. Zi H: Sensitivity analysis approaches applied to systems biology models. IET Syst Biol 2011, 5:336-346.

\section{Submit your next manuscript to BioMed Central and take full advantage of:}

- Convenient online submission

- Thorough peer review

- No space constraints or color figure charges

- Immediate publication on acceptance

- Inclusion in PubMed, CAS, Scopus and Google Scholar

- Research which is freely available for redistribution 